\title{
Functional Magnetic Resonance Imaging (FMRI)
}

\author{
C. Adharsh John \\ * B. Tech. Vishwajyothi College of Engineering
}

\begin{abstract}
People express their mental states, including emotions, thoughts, and desires, all the time through facial expressions, vocal nuances and gestures.

Functional MRI or functional Magnetic Resonance Imaging (fMRI) is a type of specialized MRI scan. It measures the haemodynamic response (change in blood flow) related to neural activity in the brain or spinal cord of humans or other animals. It is one of the most recently developed forms of neuro-imaging. Since the early 1990s, fMRI has come to dominate the brain mapping field due to its relatively low invasiveness, absence of radiation exposure, and relatively wide availability.

fMRI's might be the future technology to read your thoughts and emotions. There have been claims that fMRI can determine if you are telling the truth, what image you are looking at, and perhaps - in the future - what you are thinking about, feeling, or your intentions
\end{abstract}

\section{INTRODUCTION}

$\mathrm{T}$ he idea that regional cerebral blood flow (CBF) could reflect neuronal activity began with experiments of Roy and Sherrington at 1890 (1). This concept is the basis for all hemodynamic-based brain imaging techniques being used today. Functional MRI is a very powerful method to map brain functions with relatively high spatial and temporal resolution. In order to utilize fMRI techniques efficiently and interpret fMRI data accurately, it is important to examine underlying physiology and physics. In this article, we will discuss the signal source of the BOLD signal and improvement of BOLD fMRI techniques. Also, experimental hardware and software will be described for helping non-experts understanding MRI.

\section{WHAT IS MIND READING?}

Our mental states shape the decisions that we make, govern how we communicate with others, and affect our performance. The ability to attribute mental states to others from their behavior and to use that knowledge to guide our own actions and predict those of others is known as theory of mind or mind-reading.

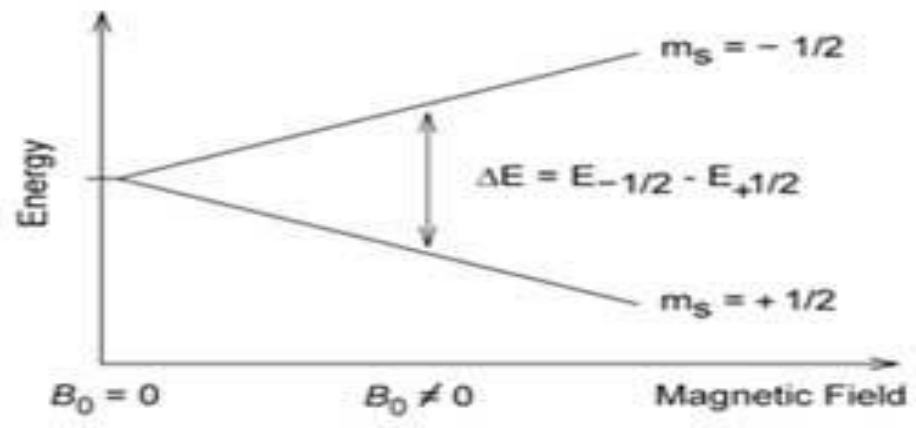

Fig 2: Relationship between energy and magnetic field

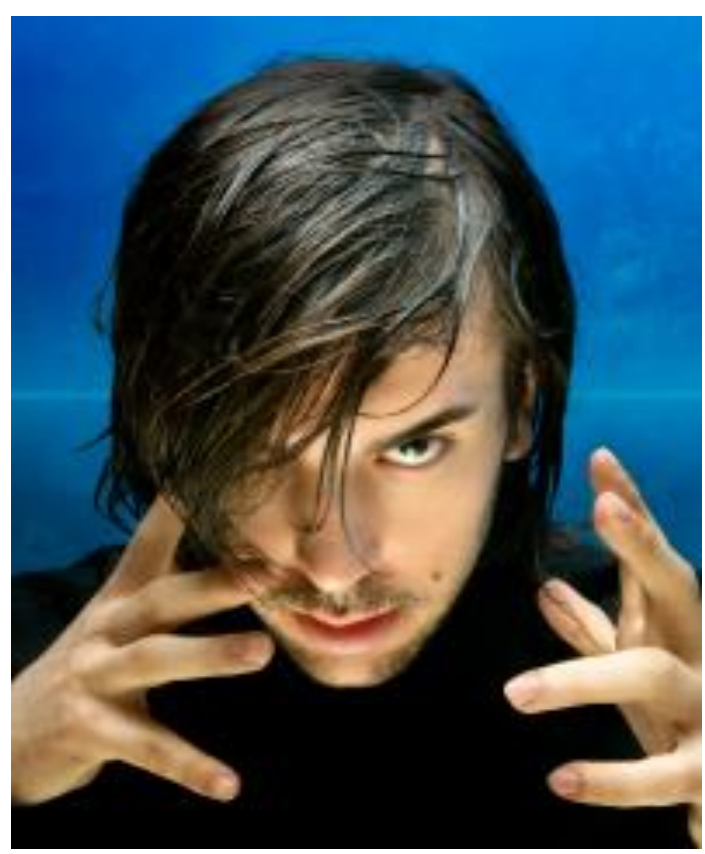

Fig1:mind reader

In scientific basis mind reading may be defined as a neuroimaging technique which allows us to detect the brain areas which are involved in a task, a process or an emotion.

\section{PRINCIPLE OF MRI}

NUCLEAR MAGNETIC RESONANCE

When a nucleus with an overall magnetic moment is placed in an external magnetic field it will either line up parallel or antiparallel (often termed spin up or spin down) with the external magnetic field, as shown below- 

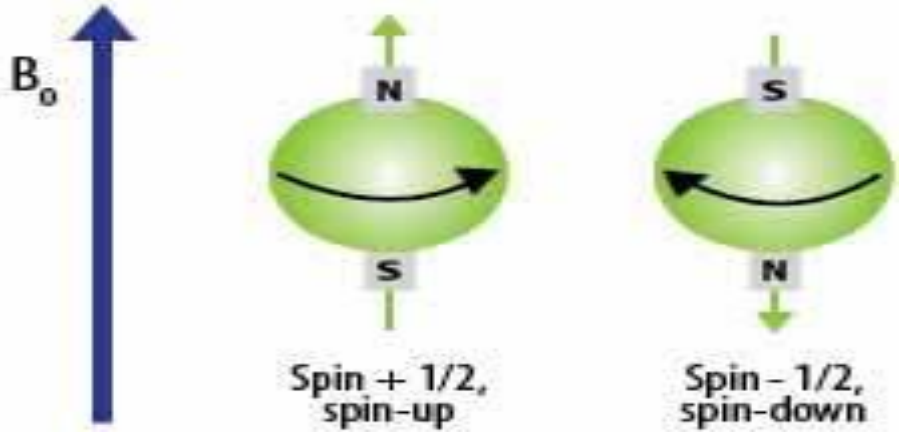

Fig3: Magnetic dipole moment

Energy splitting of nuclear spin states as a function of an external magnetic field, B0.ms $=+1 / 2$ is the spin up state and $\mathrm{ms}$ $=-1 / 2$ is the spin down state.

The difference between these two energy

Fig 2

states is proportional to the strength of the external magnetic field that the nucleus is in. As the external field increases, the energy difference between the two states increases.

The energy difference is usually related to an amount of energy resonant with a radio frequency pulse. When a radio pulse is applied to the system, it can flip the spin, causing this subatomic magnetic needle to align itself antiparallel to the external magnetic field (the higher energy state).

If a radiofrequency pulse is emitted of just the right amount of energy for the atom to absorb, the lower energy spin states will flip to the higher energy state. When the atoms go back to the lower energy state, they emit a radio frequency. When many atoms absorb and then re-emit this energy there is a detectable signal.

For regular MRI's on our body the hydrogen nuclei in water molecules are used. Our bodies are more than $72 \%$ water, which is mainly found in soft tissue. A weighting is often done between the absorbed and emitted radio frequency signals to determine the different types of tissue. Then, by using s ome powerful mathematical computations and imaging techniques, the signals observed are transformed into an image of what absorbed and released these signals.

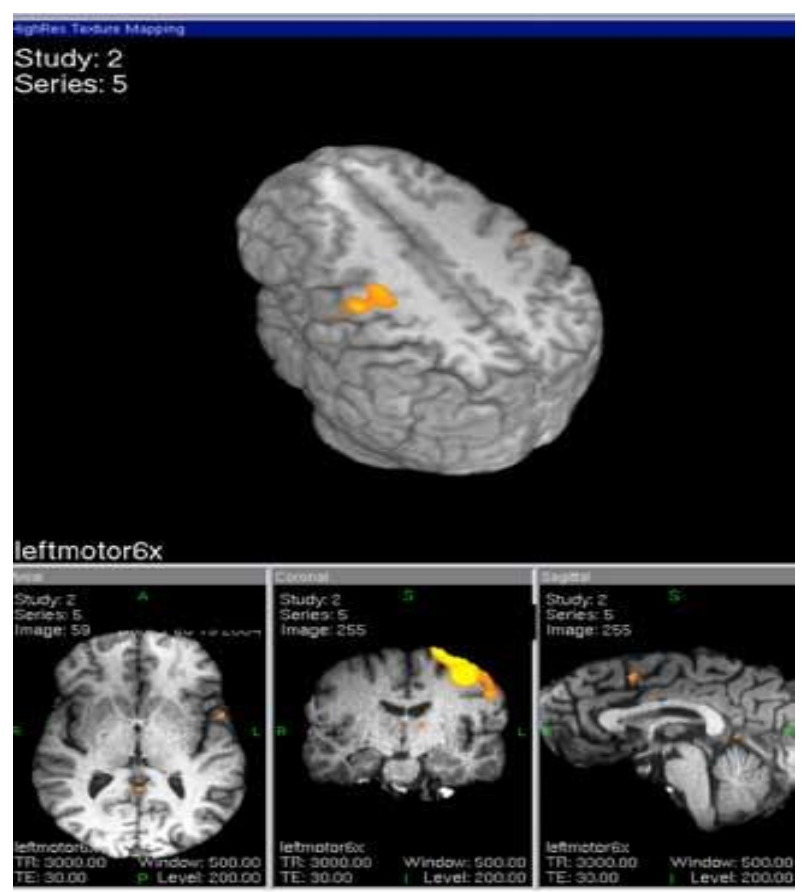

Fig 4: Brain parts affected

\section{FUNCTIONAL MRI(FMRI)}

For fMRIs the focus is on blood, blood flow, and how much oxygen is in the

Fig 5

blood. During a thought neurons are activated or are being fired in regions of the brain. These active neurons need energy and they get it from glucose supplied by blood.Oxygen rich blood has about a $20 \%$ greater magnetic strength than deoxygenated blood.

Acontrast between the oxygenated and deoxygenated blood can be observed using magnetic resonance imaging techniques.

The oxygenated blood produces the strongest signal when placed in a strong magnetic field .

Radiologists interpreting fMRI scans measure the blood flow, blood volume, and oxygen levels, which is commonly known as the Blood-Oxygen-Level-Dependent or BOLD signal. A computer processes the detected signal and maps it into 3-D images that are colored based on the measured BOLD signal .

\section{MIND READING USING FMRI}

The brain activity is mapped into cubes called voxels, which as similar to computer pixel (picture element). Each voxel (volumetric pixel element) represents tens of thousands of neurons or nerve cells. Most people have the same basic regions of the brain activated when thinking about the same object, when they have visited the same place, and when they perform or think about doing mathematical functions such as addition or subtraction. 
Radiologists cannot use fMRI to determine each neuron's function, but they can get a mapping of active parts of the brain and they are getting better at interpreting these maps.

\section{Fig6}

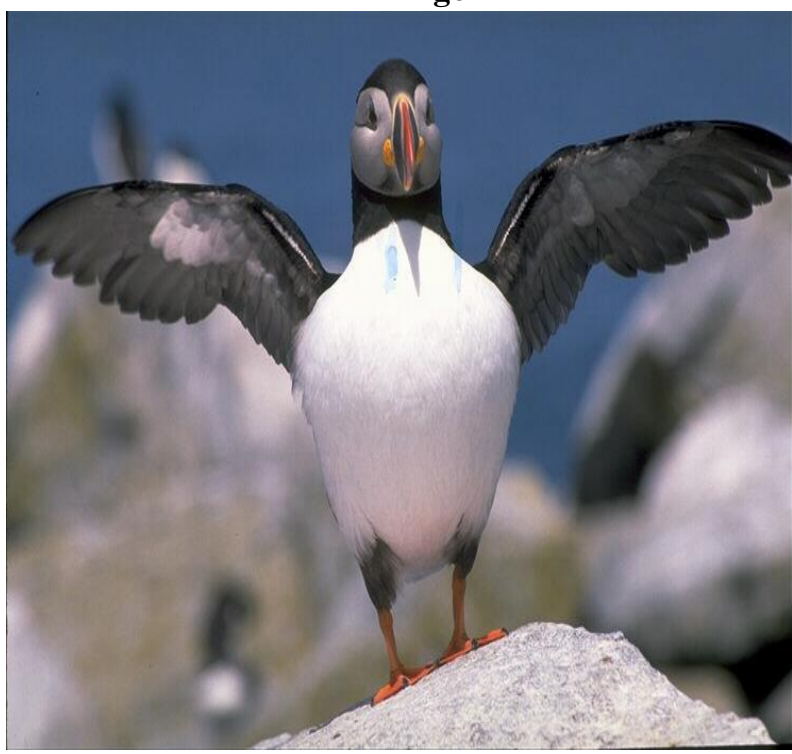

Fig \%:a bird

When you are thinking about something (let's say a bird), fMRI can show which voxels are activated (let's say voxels 3352-20 and 34-12-40). Mind reading through functional MRI is inverting this relationship: if fMRI shows you are activating voxels 33-52-20 and 34-12-40, we can assure thatyou are thinking about a bird.

\section{EXPERIMENTAL SETUP OF ANIMAL FMRI}

In order to perform MRI experiments, it is necessary to have a magnetic

\section{Fig 7}

resonanceimager, which can be obtained from MRI manufacturers.

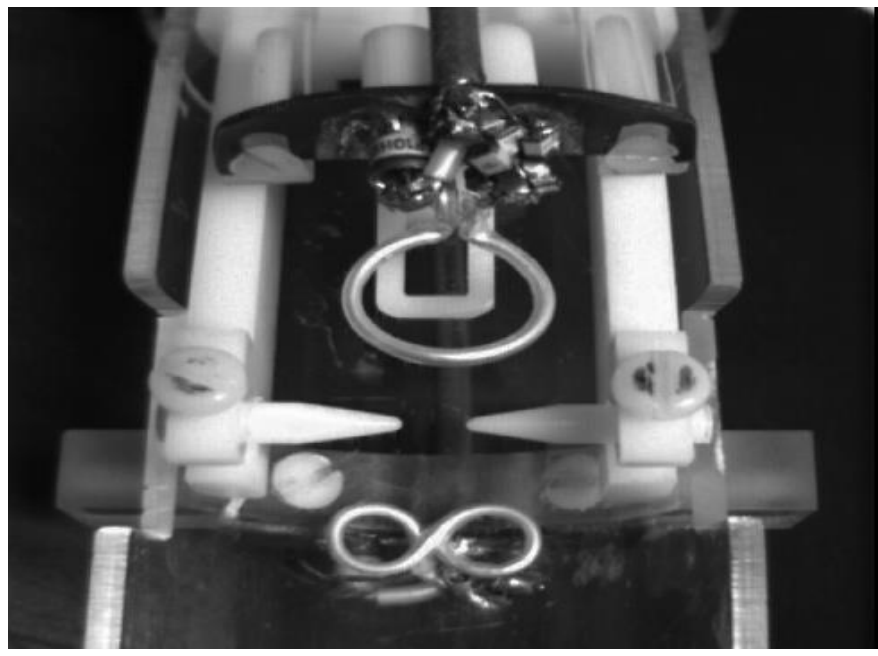

Fid 8:appartus

\section{APPAATUS REQUIRED}

An integrated MRI system consists of a magnet, gradient and shim coil(s) (often integrated), a console, radiofrequency (RF) and gradient amplifiers, and RF coils.

(i) The most expensive item is a super-conducting magnet. Strength of magnetic field (Bo) is defined as a unit of Tesla (1 Tesla ( $=10,000$ gauss) is equivalent to about 20,000 times the earth's magnetic field). Typical magnetic fields for human research range between $1.5 \mathrm{~T}$

(ii) A gradient coil set inserted into the magnet bore generates linear magnetic fields along $\mathrm{x}, \mathrm{y}$, and $\mathrm{z}$ directions.

(iii) Console consists of receivers that detect, amplify, demodulate, and digitize the MRsignals detected by the RF coil, and a set of electronics that can generate a pattern of RF and gradient pulses (which are sent to the amplifiers) for the generation of the appropriate imaging signals from the sample. A computer controls both of these processes in the console

(iv) RF coils are used to transmit RF pulses for excitation of water and detect RF signalsfrom water. For whole brain studies, a homogenous head coil should be used, while a small surface coil can be used for localized brain studies.

Anesthetized (or awake) rat is inserted into the center of magnet bore for fMRI studies. To monitoranimal conditions, end-tidal $\mathrm{CO} 2$ level, body temperature, and blood pressure are continuously monitored, and blood gases are repeatedly measured. Several changes were made in rats surroundings ,like temperature, light\&heat etc.\& different BOLD signals were obtained.

fMRI time courses of spin-echo BOLD signal in the primary somatosensory region of rat. Color indicates a cross-correlation value. Localized activation is observed at the forelimb

These datas are passed through fmri data processing softwares.fMRI data processing software can be obtained from various sources (AFNI, Brain Voyager, FIESTA?, SPM, Stimulate, etc). Processing software contain various statistical methods as well as visualization methods.

\section{APPLICATIONS OF THIS TECHNOLOGY}

Mind Controlled Wheelchair
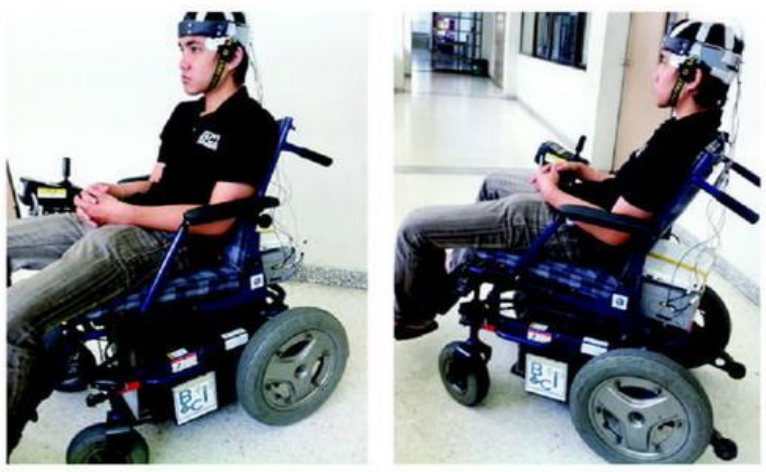

Fig 9 
A little different from the Brain-Computer Typing machine, this thing works by mapping brain waves when you think about moving left, right, forward or back, and then assigns that to a wheelchair command of actually moving left, right, forward or back.

The result of this is that you can move the wheelchair solely with the power of your mind. It will be truly helpful for the paralysed and disabled people.

\section{WORLD WITHOUT INPUT DEVICES}
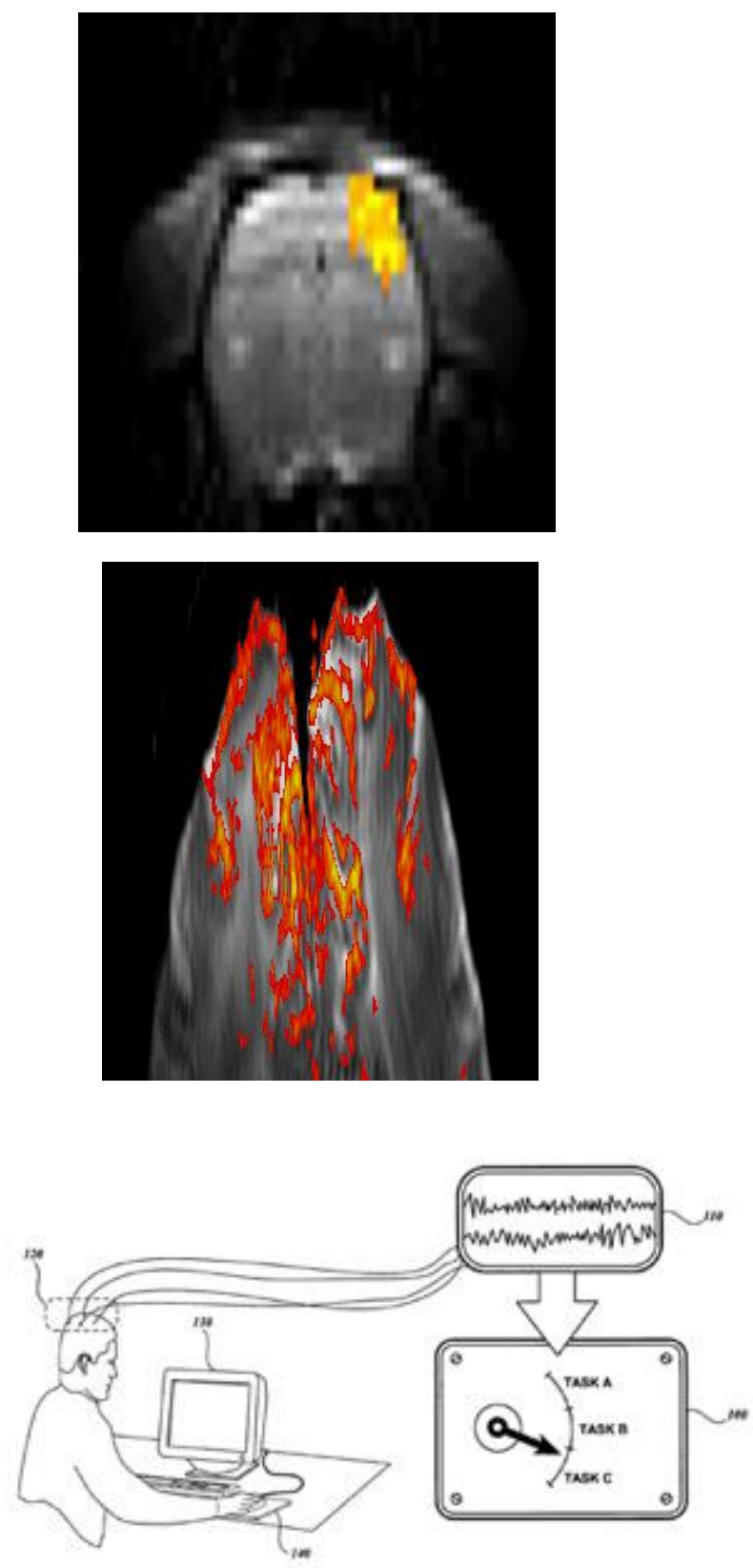

Fig 10 :brain features in a mri scan report

Huge time is spent in input data to computers. By using this technology input can be given directly from mind to system,Leading to tremendous changes in industrial,health,\& educational fields.

\section{IN COMMUNICATION FIELD}

A future where we are surrounded with mobile phones, cars and online services that can read our minds and react to our moods. Even we can drive cars without streeing.

\section{CRIMINAL BRAINMAPPPING \& LIE DETECTOR}

Development of this technology will become an helpful aid in reading intentions of criminals on scientific basis and to investigate crimes.

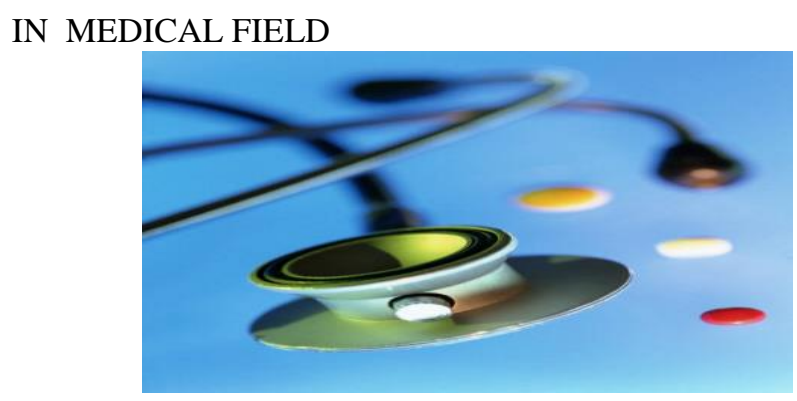

Fig 12: General

Physicians perform fMRI to:

Examine the anatomy of the brain.

-Determine precisely which part of the brain is handling critical functions such as thought, speech, movement and sensation, which is called brain mapping.

-Help assess the effects of stroke, trauma or degenerative disease (such as Alzheimer's) on brain function.

- Monitor the growth and function of brain tumours. Guide the planning of surgery, radiation therapy, or other surgical treatments for the brain.

\section{ADVANTAGES \& DEMERITS}

\section{ADVANTAGES}

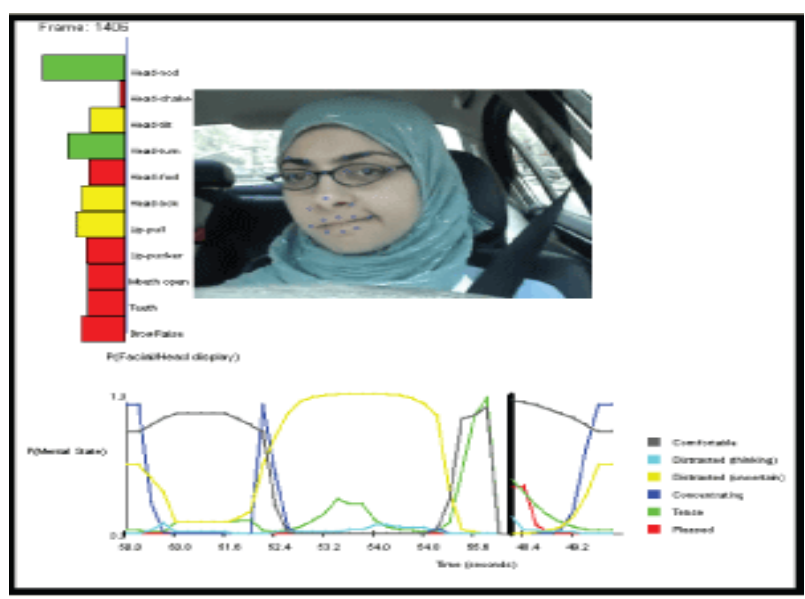

Fig 12: report 
fMRI and MRI machines are harmless with virtually no side effects. However they are not for those who have claustrophobia or any metal pins or rods in their bodies.

They are effective to determine areas of thought, brain function, and the health of brain tissue, as well as analyzing emotion.

Short acquisition time of images

Mapping of complex functions (emotions, motor control, specialized language functions,...) is possible.

\section{DEMERITS}

\section{TAPPING OF BRAINS FOR FUTURE CRIMES}

Freedom of right to think any time, any thing at any where gets destroyes. Chances of misusing this technology to tap one's thoughts will lead to danger

ONLY SIMPLEX MODE OF COMMUNICATION POSSIBLE

Only signals from brain can be detected.Signals to brain cannot be propagated.

\section{CALIBRATION VARY FROM PERSON TO PERSON}

Individual brains differ, so scientists need to study a subject's patterns before they can train a computer to identify those patterns or make predictions.

APPARATUS REQUIRED ARE COSTLIER \& HUGE.

\section{CONCLUSION}

Functional magnetic resonance images (fMRIs) are becoming popular in both the consumer and research arenas. Big businesses use fMRIs to determine consumer preferences and which movie scenes viewers prefer. Smaller businesses utilize the machines as lie detector tests. However, the technique of fMRI is being most utilized for clinical and basic research by many different professions.

Imaging brain activity is a source of data that provides insights into individual characteristics.

This technology is going to be a mojor tool for scientist's in the development of next generation computers working on artificial intelligence.

\section{REFERENCES}

[1] http://www.ebme.co.uk/arts/links/fmri.php

[2] Principles of Functional MRI(pdf) Seong-Gi Kim, Ph.D. Department of Neurobiology, University of Pittsburgh Medical School

[3] http://www.physicscentral.com/fmri.htm

[4] A Primer on MRI and Functional MRI(pdf) (version 2.1, 6/21/01) Douglas C. Noll, Ph.D. Departments of Biomedical Engineering and Radiology University of Michigan, Ann Arbor, MI 48109-2125

\section{AUTHORS}

First Author - C.Adharsh john, Btech : Vishwajyothi college of Engineering, Mail:adhajohn@gmail.com, Phno:91-9633315206 